\title{
Los Prejuicios y las Actitudes para el Aprendizaje del Aymara de los Docentes de la Universidad Nacional Jorge Basadre Grohmann- Tacna
}

\author{
Prejudices and Attitudes to Learning of Aymara in Professors of \\ Universidad Nacional Jorge Basadre Grohmann - Tacna
}

\author{
${ }^{1}$ Ernesto Pino Nina
}

\begin{abstract}
RESUMEN:
Esta investigación aborda los prejuicios y las actitudes lingüísticas para el aprendizaje de la lengua aymara. Persigue como objetivo el determinar el nivel de ambas variables y la correlación entre ellas.

La metodología empleada para la obtención de datos fueron dos encuestas, las que se aplicaron a una muestra de 93 docentes universitarios. Mediante el test de Chi-Cuadrado y el coeficiente Rho de Spearman se comprobó que existe una relación directa significativa entre las dos variables. La mayoria de los docentes de la Universidad Nacional Jorge Basadre Grohmann de las 32 carreras profesionales, se ubicaron en el nivel poco prejuicio; y en cuanto a las actitudes, más de la mitad se inclinaron hacia lo favorable para el aprendizaje del aymara.
\end{abstract}

Palabras clave: Prejuicios, actitudes, aprendizaje del aymara.

\section{ABSTRACT:}

This research focuses on the attitudes and prejudices to the learning of the Aymara language whose objective is to determine the level of both variables and the correlation between them.

The methodology used in order to obtain the data collection was two surveys with the conditions applied to a sample of 93 university professors. Using the chi square test and the Spearman coefficient Rho it was found that there is a direct relationship between the two variables. Most of the professors from Jorge Basadre Grohmann University National of 32 careers, were placed in the level of little prejudice; and as to the attitudes, more than half tended to be favorable to the learning of Aymara.

Key Words: Prejudices, attitudes, learning of Aymara.

\footnotetext{
Magister Scientiae en Tecnología Educativa. Lic. en Educación - Lengua y Literatura, docente de la Facultad de Educación, Comunicación y Humanidades/Universidad Nacional Jorge Basadre Grohmann de Tacna. Perú.
} 


\section{INTRODUCCIÓN}

La Región Tacna cuenta con un patrimonio lingüístico que es el aymara. Fueron los jesuitas de Juli quienes bautizaron la lengua colla como aymara, pero con la salvedad de que esta región era multiétnica (CerrónPalomino, 2000). Desde la época de la Colonia esta lengua quedó marginada en todas las funciones lingüísticas con la imposición del español.

La familia aymara está constituida por el aymara central (Ibid) y el collavino o sureño (norteño, intermedio y sureño). En el Perú se cuenta con 421380 aimarahablantes de ambas familias; en Chile, 14 924; en Bolivia, donde está la mayor concentración de hablantes de esta lengua, con 1 millón 600000 ; en total, aproximadamente 2044388 .

Godenzzi (1998) por su parte indica que en el Perú se cuenta con 300000 aimarahablantes, según el trabajo de campo realizado en 1997. Y Gamero (2000) refiere que en el departamento de Tacna la lengua aymara cuenta con 40411 hablantes distribuidos de la siguiente manera: En la provincia de Tacna, 35 602; Candarave, 1 838; Tarata, 1 751; y Jorge Basadre, 1220.

Según Tusón (2003: 27), el prejuicio lingüístico es una desviación de la racionalidad que, casi siempre, toma la forma un juicio de valor o bien sobre una lengua (o alguna de sus características), o bien sobre los hablantes de una lengua (en tanto que hablantes). Y se trata de un prejuicio generalmente dictado por la ignorancia o por la malevolencia, ajustado a estereotipos maniqueos y dictado por la desazón que producen todas aquellas cosas y personas que son diferentes a uno. Los prejuicios son un lastre terrible que trunca todo proyecto de promoción social de educación intercultural bilingüe; de allí que sea importante estudiarlos a fin de coadyuvar en la armonía, paz y dignidad de las lenguas en el mundo, tal como refiere Moreno (2002) y el Comité de Seguimiento de la Declaración Universal de los Derechos Lingüísticos (1998).

Las actitudes lingüísticas son un conjunto de creencias, sentimientos y tendencias hacia una lengua, dialecto o manera de hablar (Martínez y Mora, 2008). La identidad lingüística, el constructo abstracto, se manifiesta principalmente a través de las actitudes lingüísticas, las que están conformadas por factores cognitivos, afectivos y conductuales. Por otra parte, el grado de vitalidad de una lengua depende mucho de estas actitudes (Escobar, en Adelaar, 2011: 126), aparte de si la lengua goza de prestigio y de lealtad de sus hablantes, del apoyo institucional con políticas lingüísticas, entre otros factores.

El presente artículo es parte de una investigación que se realizó en el Consejo de Investigación; por ello la información que se presenta es sintética. El problema fue el siguiente: ¿Cómo se relacionan los prejuicios y las actitudes de los docentes de la Universidad Nacional Jorge Basadre Grohmann para el aprendizaje de la lengua aymara? Se consignó como hipótesis general lo siguiente: Los prejuicios y las actitudes para el aprendizaje de la lengua aymara no guardan una relación directa.

\section{MATERIALES Y MÉTODOS}

La investigación es básica y se caracterizó por presentar un diseño mixto. Por su ubicación en el tiempo, es una investigación transversal; por la forma en que se realiza, un diseño no experimental.

La población objeto de estudio fueron los docentes (546) nombrados y contratados de ambos sexos, de las siete facultades y 32 carreras profesionales de la Universidad Nacional Jorge Basadre Grohmann. A partir de esta cantidad, se obtuvo la muestra aleatoria por facultades y con criterio de inclusión de los participantes: 93 .

Una de las variables de estudio fue los prejuicios, cuyos indicadores fueron los juicios y las creencias; la otra, las actitudes, las que tuvo tres indicadores: nivel de tratamiento social, de preferencia lingüística y el de reacción subjetiva.

Para la recopilación de la información, se empleó la técnica de la encuesta, la cual fue una adaptación a la de Amorrortu \& al. (2009). Dado que la investigación es de tipo no aplicada, no se consignaron equipos e instrumentos para la ejecución de la investigación. Pero se podría citar algunos equipos auxiliares como la Laptop, impresora, calculadora, concretamente.

Para el procesamiento de la información se utilizó el software estadístico SPSS, versión 17. Para el análisis de la información se empleó la estadística descriptiva y la inferencial; en el primer caso, la descripción de cuadros de frecuencias porcentuales y figuras de barras dobles; en el segundo caso, para la prueba de hipótesis, el Test de Chi Cuadrado y Rho de Spermann.

\section{Objetivos}

Determinar cómo se relacionan los prejuicios y las actitudes de los docentes de la Universidad Nacional Jorge Basadre Grohmann para el aprendizaje de la lengua aymara.

Determinar el nivel de los prejuicios y actitudes para el aprendizaje del aymara.

\section{RESULTADOS}

Luego de haber procesado la información obtenida, se observó que el $94,6 \%$ de los docentes de la Universidad Nacional Jorge Basadre Grohmann tuvo como lengua materna el español, de los cuales el $36,6 \%$ aprendió el inglés, y el $31,2 \%$ son monolingües hispanos. Por otra parte, el $3,2 \%$ aprendió primero el aymara; y el $2,2 \%$, el quechua. El 54,8\% declaró que ningún familiar suyo habla el aymara; el porcentaje restante $(45,2 \%)$ afirmó tener algún parentesco, ya sean los abuelos o los padres. Por la hegemonía de la cultura occidental, hay un desmedro de las lenguas y culturas originarias en la práctica, lo cual podría explicar que a pesar de contar con un porcentaje considerable de entorno familiar aymara, los docentes encuestados no hablen dicha lengua. 
3.1 Nivel de prejuicios lingüísticos.- Para determinar dicho nivel, se presentó a los encuestados 16 preguntas en un cuestionario. He aquí algunas de ellas. En lo referente a la creencia de que "El aymara sigue siendo una lengua de serranos pobres", los docentes universitarios respondieron de la siguiente manera: El $50,5 \%$ se mostró muy en desacuerdo; el $39,8 \%$, en desacuerdo; el 6,5\%, ni de acuerdo ni en desacuerdo; el $2,2 \%$, de acuerdo; y el $1,1 \%$, muy de acuerdo. Este rechazo mayoritario a esta idea racista implica que la mayoría de los encuestados se muestre favorable hacia la lengua y cultura aymaras.

Ante la pregunta "Que haya muchas lenguas como el aymara en el Perú, perjudica el desarrollo del país", los docentes universitarios se mostraron del siguiente modo: El 51,6\%, en desacuerdo; el 31,2\%, muy en desacuerdo; el 9,7\%, ni de acuerdo ni en desacuerdo; el $7,5 \%$, de acuerdo; y ningún porcentaje muy de acuerdo. Hablar varias lenguas ayuda a superar las ideas etnocentristas del lenguaje y propicia una actitud comprensiva a lo diferente; por ello, coincidentemente los resultados más saltantes niegan el supuesto perjuicio al desarrollo nacional; solo el 7,5\% del total de docentes universitarios se mostró prejuiciosos hacia lo señalado.

Además, ante la idea de que "No es significativo para el Perú recuperar el aymara", los docentes universitarios

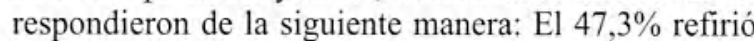
estar en desacuerdo; el $24,7 \%$, muy en desacuerdo; el $11,8 \%$, ni de acuerdo ni en desacuerdo; el $12,9 \%$, de acuerdo; y el 3,2\%, muy de acuerdo. Mayoritariamente, los docentes encuestados, al rechazar con sus respuestas, dan a entender que es significativo para el Perú recuperar el aymara; es más, lo idóneo hubiera sido que después de la Independencia nacional se volviese a los milenarios legados lingüístico-culturales y que no se siguiese prolongando hasta hoy el colonialismo mental y todo su actuar. En otros términos, prevaleció en tales percepciones el concepto de la interculturalidad.

Después de haber constatado varios ítem, se estableció el nivel de prejuicios respecto al aprendizaje del aymara de los docentes de la Universidad Nacional Jorge Basadre Grohmann: El 51,6\%, poco prejuicioso; el $29,0 \%$, nada prejuicioso; el $14,0 \%$, regularmente prejuicioso; el $5,4 \%$, prejuicioso; y ningún porcentaje, muy prejuicioso. El 80,6\% de los encuestados adoptó un nivel favorable hacia el aprendizaje del aymara y el $5,4 \%$, un nivel desfavorable ante ello, a lo que se suman los del nivel regular en un $14,0 \%$. De todos modos estos últimos datos indican que se tendrá que concientizar para erradicar cualquier forma de discriminación lingüística (Ver Tabla $\left.N^{\circ} 1\right)$.

3.2. Nivel de actitudes lingüísticas.- Para precisar el nivel de actitudes de los docentes de la Universidad Nacional Jorge Basadre Grohmann de Tacna - Perú, se preparó un cuestionario de 12 preguntas. Entre las más relevantes se puede considerar las siguientes:

Referente a la idea de que "Los y las niñas de la Región Tacna deben aprender el aymara", los docentes universitarios respondieron de la siguiente manera: El $48,4 \%$, de acuerdo; el $11,8 \%$, muy de acuerdo; el $25,8 \%$, ni de acuerdo ni en desacuerdo; el 7,5\%, en desacuerdo; y el $6,5 \%$, muy en desacuerdo. Por lo visto, el $60,2 \%$ de los encuestados se mostraron a favor de que los niños de la Región Tacna deban aprender el aymara; y contrariamente, el 14,0\% sostuvo que no; en tanto que el $25,8 \%$ se mostró dubitativo.

Ante la pregunta de que "No hay que dejar morir el aymara", los docentes universitarios contestaron de la siguiente manera: El 48,4\%, de acuerdo; el 28,0\%, muy de acuerdo; el 16,1\%, ni de acuerdo ni en desacuerdo; el $6,5 \%$, en desacuerdo; y el 1,1\%, muy en desacuerdo. En otros términos, el $76,4 \%$ de docentes universitarios se mostró favorable con que no hay que dejar morir el aymara; estos resultados dan la esperanza para la vigencia de la lengua indicada. Contrariamente, el 7,6\% asumió una actitud negativa ante ello; en tanto que el $16,1 \%$ se mostró vacilante.

TABLA N 1

NIVEL DE PREJUICIOS DE LOS DOCENTES DE LA UNJBG PARA EL APRENDIZAJE DEL AYMARA (Total) Tacna -2011

\begin{tabular}{|c|c|c|c|c|c|c|c|c|c|c|c|c|c|c|c|c|}
\hline \multirow{4}{*}{$\begin{array}{l}\text { Nivel } \\
\text { Muy } \\
\text { prejuic ioso }\end{array}$} & \multicolumn{14}{|c|}{ FACULTADES } & \multirow{2}{*}{\multicolumn{2}{|c|}{ T otal }} \\
\hline & \multicolumn{2}{|c|}{$\begin{array}{l}\text { ING. CIVIL. } \\
\text { ARQ Y Y } \\
\text { GEOTEC. }\end{array}$} & \multicolumn{2}{|c|}{$\begin{array}{c}\text { ED_, COM Y } \\
\text { HUM. }\end{array}$} & \multicolumn{2}{|c|}{$\begin{array}{l}\text { CS JUR Y } \\
\text { EMPR. }\end{array}$} & \multicolumn{2}{|c|}{ ING. } & \multicolumn{2}{|c|}{ CS. AGROP. } & \multicolumn{2}{|c|}{ cs } & \multicolumn{2}{|c|}{$\begin{array}{l}\text { CS.DE LA } \\
\text { SALUD }\end{array}$} & & \\
\hline & 7 & $\%$ & $f$ & $\%$ & f & $\%$ & f & $\%$ & i & $\%$ & $f$ & $\%$ & i & $\%$ & $f$ & $\%$ \\
\hline & 0 & 0 & 0 & 0 & 0 & 0 & 0 & 0 & 0 & 0 & 0 & 0 & 0 & 0 & 0 & 0 \\
\hline Prejutetoso & 0 & $0 \%$ & 0 & $.0 \%$ & 3 & $30.0 \%$ & 0 & $.0 \%$ & 0 & $.0 \%$ & 1 & $6.7 \%$ & 1 & $4.5 \%$ & 5 & $5.4 \%$ \\
\hline $\begin{array}{l}\text { Regu larm ente } \\
\text { prejuic ioso }\end{array}$ & 4 & $50.0 \%$ & 0 & $.0 \%$ & 2 & $20.0 \%$ & 1 & $10.0 \%$ & 2 & $15.4 \%$ & 1 & $6.7 \%$ & 3 & $136 \%$ & 13 & $14.0 \%$ \\
\hline p rejuic io so & 3 & $37.5 \%$ & 8 & $533 \%$ & 3 & $30.0 \%$ & 5 & $50.0 \%$ & 8 & $61.5 \%$ & 7 & $46.7 \%$ & 14 & $636 \%$ & 48 & $51.6 \%$ \\
\hline $\begin{array}{l}\mathrm{Nada} \\
\text { prejuic io so }\end{array}$ & 1 & $125 \%$ & 7 & $46.7 \%$ & 2 & $20.0 \%$ & 4 & $40.0 \%$ & 3 & $23.1 \%$ & 6 & $40.0 \%$ & 4 & $182 \%$ & 27 & $29.0 \%$ \\
\hline Total 1 & 8 & $\begin{array}{c}100.0 \\
\%\end{array}$ & 15 & $\begin{array}{c}100,0 \\
\%\end{array}$ & 10 & $\begin{array}{l}100.0 \\
\%\end{array}$ & 10 & $\begin{array}{c}100.0 \\
\%\end{array}$ & 13 & $\underset{\%}{100.0}$ & 15 & $\begin{array}{c}100.0 \\
\%\end{array}$ & 22 & $\begin{array}{c}100,0 \\
\%\end{array}$ & 93 & 100.0 \\
\hline
\end{tabular}


Respecto a la idea de que "En general, se debe aprender el aymara en el hogar y en todos los niveles educativos", los docentes universitarios respondieron de la siguiente manera: El 45,2\%, de acuerdo; el 8,6\%, muy de acuerdo; el 28,0\%, ni de acuerdo ni en desacuerdo; el 8,6\%, en desacuerdo; y el 9,7\%, muy en desacuerdo. Por lo visto, la mayoría de los encuestados asumieron una actitud positiva ante ello.

Después de haber examinado varios ítem en torno a la segunda variable, se precisó el nivel de las actitudes para el aprendizaje del aymara desde la perspectiva de los docentes de la Universidad Nacional Jorge Basadre Grohmann y es como se detalla: El 51,6\%, favorable; el $8,6 \%$, muy favorable; el $22,6 \%$, regularmente favorable; el $16,1 \%$, poco favorable; y el $1,1 \%$, nada favorable. Como se podrá apreciar, lo poco favorable y nada favorable no supera el $17,2 \%$ de los encuestados, lo que significa que es viable el fomento de la enseñanzaaprendizaje de la lengua aymara según la actitud de los docentes basadrinos (Ver Tabla No 2 ).

A partir de estos resultados totales de las dos variables, mediante la prueba de Chi-Cuadrado y el coeficiente Rho de Spearman, se pudo comprobar que los prejuicios y las actitudes para el aprendizaje de la lengua aymara guardan una relación directa en los docentes de la mencionada universidad en el año 2011, para un nivel de significancia de $5 \%$.

\section{DISCUSIÓN}

En lo que respecta a los prejuicios lingüísticos, en un estudio realizado por Pino (2011), se pudo comprobar que prevaleció el nivel poco prejuicioso en los alumnos de la Facultad de Educación, Comunicación y Humanidades de la Universidad Nacional Jorge Basadre Grohmann para el aprendizaje de la lengua aymara, para un nivel de significancia de 5\%. Ahora, con este último estudio que se presenta, se pudo comprobar también que existe el mismo nivel en los docentes de la misma universidad para el aprendizaje de la misma lengua, para un nivel de significancia del $5 \%$. Como se podrá apreciar, ambos grupos de estudio se ubican en el nivel poco prejuicioso, lo cual significa que a pesar de ello se tiene que concientizar a los agentes educativos a fin de respetar y promover esta lengua que es patrimonio de toda la humanidad.

En torno a las actitudes lingüísticas, en el mismo estudio del autor antes mencionado se pudo comprobar que existe un nivel favorable de actitudes en los alumnos universitarios para el aprendizaje de la lengua aymara, para un nivel de significancia de 5\%. En el presente estudio, se pudo determinar que existe también un nivel favorable de actitudes en los docentes para el aprendizaje de tal lengua, para un nivel de significancia de $5 \%$. Por lo visto, en ambos grupos de muestra prevaleció el nivel favorable para el aprendizaje del aymara, lo cual debe considerarse en las innovaciones curriculares en todas las carreras profesionales como en otros niveles educativos.

TABLA N 2

NIVEL DE ACTITUDES DE LOS DOCENTES PARA EL APRENDIZAJE DEL AYMARA (Total) Tacna -2011

\begin{tabular}{|c|c|c|c|c|c|c|c|c|c|c|c|c|c|c|c|c|}
\hline \multirow{3}{*}{ Actitud } & \multicolumn{14}{|c|}{ FACULTADES } & \multirow{2}{*}{\multicolumn{2}{|c|}{ Total }} \\
\hline & \multicolumn{2}{|c|}{$\begin{array}{c}\text { ING. CIVIL, } \\
\text { ARQ. Y } \\
\text { GEOTEC. }\end{array}$} & \multicolumn{2}{|c|}{$\begin{array}{c}\text { ED., COM. Y } \\
\text { HUM. }\end{array}$} & \multicolumn{2}{|c|}{$\begin{array}{l}\text { CS. JUR. Y } \\
\text { EMPR. }\end{array}$} & \multicolumn{2}{|c|}{ ING. } & \multicolumn{2}{|c|}{ CS. AGROP. } & \multicolumn{2}{|r|}{$\mathrm{CS}$. } & \multicolumn{2}{|c|}{$\begin{array}{l}\text { CS. DE } \\
\text { LA } \\
\text { SALUD }\end{array}$} & & \\
\hline & $\mathrm{f}$ & $\%$ & f & $\%$ & $f$ & $\%$ & f & $\%$ & f & $\%$ & f & $\%$ & $\mathrm{f}$ & $\%$ & $f$ & $\%$ \\
\hline Nada favorable & 0 & $.0 \%$ & 0 & $.0 \%$ & 0 & $.0 \%$ & 1 & $10.0 \%$ & 0 & $.0 \%$ & 0 & $.0 \%$ & 0 & $.0 \%$ & 1 & $1.1 \%$ \\
\hline Poco favorable & 6 & $75.0 \%$ & 0 & $.0 \%$ & 5 & $50.0 \%$ & 1 & $10.0 \%$ & 1 & $7.7 \%$ & 2 & $13.3 \%$ & 0 & $.0 \%$ & 15 & $16,1 \%$ \\
\hline $\begin{array}{l}\text { Regularmente } \\
\text { favorable }\end{array}$ & 0 & $.0 \%$ & 2 & $13.3 \%$ & 4 & $40.0 \%$ & 0 & $.0 \%$ & 5 & $38.5 \%$ & 2 & $13.3 \%$ & 8 & $\begin{array}{c}36.4 \\
\%\end{array}$ & 21 & $22.6 \%$ \\
\hline Favorable & 1 & $12.5 \%$ & 10 & $66.7 \%$ & 1 & $10.0 \%$ & 8 & $80.0 \%$ & 7 & $53.8 \%$ & 10 & $66.7 \%$ & 11 & $\begin{array}{c}50.0 \\
\%\end{array}$ & 48 & $51.6 \%$ \\
\hline Muy favorable & 1 & $12.5 \%$ & 3 & $20.0 \%$ & 0 & $.0 \%$ & 0 & $.0 \%$ & 0 & $.0 \%$ & 1 & $6.7 \%$ & 3 & $\begin{array}{c}13.6 \\
\%\end{array}$ & 8 & $8.6 \%$ \\
\hline Total & 8 & $100.0 \%$ & 15 & $100.0 \%$ & 10 & $100.0 \%$ & 10 & $100.0 \%$ & 13 & $100.0 \%$ & 15 & $100.0 \%$ & 22 & $\begin{array}{c}100 . \\
0 \%\end{array}$ & 93 & 100.0 \\
\hline
\end{tabular}

Fuente: Elaboración propia a partir de la encuesta 2 


\section{CONCLUSIONES}

La investigación ha permitido arribar a las siguientes conclusiones:

En el año 2011, el $94,6 \%$ de los docentes de la Universidad Nacional Jorge Basadre Grohmann sostuvieron tener como primera lengua el español; el aymara, el $3,2 \%$; y el quechua, $2,2 \%$. De los 93 encuestados, el $45,2 \%$ afirmó tener algún parentesco aymara, y se ha podido verificar que el aprendizaje de esta lengua ha menguado notablemente generación tras generación, lo cual es un indicador de que esta lengua está perdiendo su vitalidad por falta de hablantes.

Los encuestados rechazaron mayoritariamente los prejuicios como los siguientes: El aymara sigue siendo una lengua de serranos pobres; que haya muchas lenguas como el aymara en el Perú, perjudica el desarrollo del país; no es significativo para el Perú recuperar el aymara. En suma, un poco más de la mitad de los docentes universitarios $(51,6 \%)$ se ubicaron en el nivel poco prejuicioso, seguido de los nada prejuiciosos.

Referente a las actitudes para el aprendizaje del aymara, la mayoría se mostró favorable a que los niños y las niñas de la Región Tacna deben aprender esta lengua; que no hay que dejarla morir; y en general se debe aprender el aymara en el hogar y en todos los niveles educativos. En suma, existe un nivel favorable de actitudes para el aprendizaje de la lengua aymara, seguido de los regularmente favorable, en los docentes de la Universidad Nacional Jorge Basadre Grohmann de Tacna.

Por otra parte, existe una relación directa entre los prejuicios y las actitudes para el aprendizaje del aymara, con un nivel de significación del $5 \%$.

Finalmente, se sugiere que en todos los niveles educativos de la Región Tacna se debe propiciar el aprendizaje de esta lengua. Además, la comunidad lingüistica aymara debe enseñar a sus hijos con mayor lealtad e identidad cultural la lengua que le es propia y original.

\section{REFERENCIAS BIBLIOGRÁFICAS}

ADELAR, W. et al. (2011) [Editores]. Estudios sobre lenguas andinas y amazónicas. Homenaje a Rodolfo Cerrón Palomino. Lima: Fondo Editorial de la Pontificia Universidad Católica del Perú, $476 \mathrm{pp}$.

AMORRORTU, E., ORTEGA, A., IDIAZABAL, I. y BARREÑA, A. (2009). Actitudes y prejuicios de los castellanohablantes hacia el euskera. Vitoria-Gasteiz: Gobierno Vasco, 354 pp.
CERRÓN-PALOMINO, R. (2000). Lingüistica aimara. Cusco: Centro de Estudios Regionales Andinos "Bartolomé de Las Casas", 405 pp.

COMITÉ DE SEGUIMIENTO DE LA DECLARACIÓN UNIVERSAL DE DERECHOS LINGÜÍSTICOS (1998). Declaración universal de los derechos lingüisticos. (PDF), Institut d'Edicions de la Diputación de Barcelona, Barcelona, abril de 1998. Disponible en: http://www.linguisticdeclaration.org/versions/espanyol.pdf [Consultada: 8 de febrero del 2011].

GAMERO, E. (2000). Wiñaya aymara aru/ Eterna voz aymara. Tacna: "El Tigre", $210 \mathrm{pp}$.

GODENZZI, J. (1998). Cultura de paz, bilingüismo e interculturalidad. En el boletín Coiné, Departamento Académico de Humanidades - Universidad del Pacífico, Año I, N 2, Lima, agosto de 1998. En: http://www.up.edu.pe/coine/Boletin2/TRASFON DO.html, [Consultada: 29 de setiembre del 2009].

MARTINEZ, H. y MORA, E. (2008). La identidad lingüistica y los trastornos del habla [Online]. Boletin de Lingüistica, Vol. 20, N 29, Págs. 85-101. Caracas, jun. 2008. Disponible en:

http://www.scielo.org.ve/scielo.php?script=sci_ar $\mathrm{t} t \mathrm{e} x \mathrm{t} \& \mathrm{p} \mathrm{i} \mathrm{d}=\mathrm{S} 0798$. $97092008000100004 \& \operatorname{lng}=\mathrm{es} \& \mathrm{nrm}=$ is o [Consultada el 12 enero del 2011].

MORENO, J. (2002). La dignidad e igualdad de lenguas. Critica de la discriminación lingüística. Primera Edición 2000. Madrid: Alianza Editorial, 316 pp.

PINO, E. (2011) [Tesis]. Los prejuicios y las actitudes para el aprendizaje de la lengua aymara de los alumnos de la Facultad de Humanidades, Universidad Nacional Jorge Basadre Grohmann, año 2011. Tacna: Universidad Nacional Jorge Basadre Grohmann, Escuela de Posgrado, $228 \mathrm{pp}$.

TUSON, J. (2003). Los prejuicios lingüisticos. Segunda Edición. Barcelona: Octaedro, 127 pp.

\section{Correspondencia:}

Ernesto Pino Nina

Cel. 952632915

Asoc. Viv. Villa la Agronómica Mz N Lte 6.

Ciudad Universitaria Fundo "Los Granados"

Av. Miraflores s/n. Tacna - Perú

erpinonina33@hotmail.com 